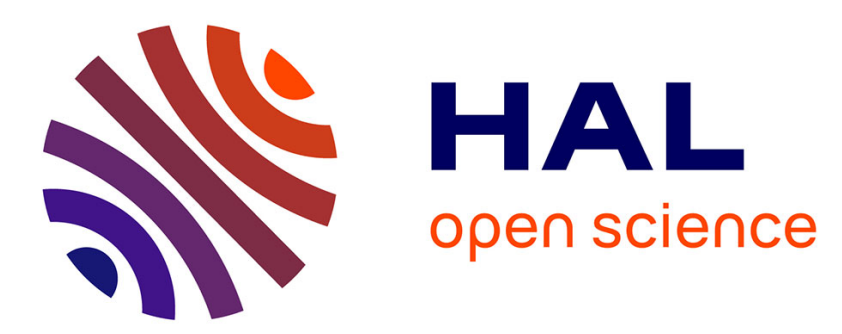

\title{
On the feasibility of interference alignment in ultra-dense millimeter-wave cellular networks
}

\author{
Jian Song, Lam Thanh Tu, Marco Di Renzo
}

\section{To cite this version:}

Jian Song, Lam Thanh Tu, Marco Di Renzo. On the feasibility of interference alignment in ultradense millimeter-wave cellular networks. 2016 50th Asilomar Conference on Signals, Systems and Computers, Nov 2016, Pacific Grove, United States. 10.1109/ACSSC.2016.7869557 . hal-01880134

\section{HAL Id: hal-01880134 \\ https://hal.science/hal-01880134}

Submitted on 16 Jul 2020

HAL is a multi-disciplinary open access archive for the deposit and dissemination of scientific research documents, whether they are published or not. The documents may come from teaching and research institutions in France or abroad, or from public or private research centers.
L'archive ouverte pluridisciplinaire HAL, est destinée au dépôt et à la diffusion de documents scientifiques de niveau recherche, publiés ou non, émanant des établissements d'enseignement et de recherche français ou étrangers, des laboratoires publics ou privés. 


\title{
On the Feasibility of Interference Alignment in Ultra-Dense Millimeter-Wave Cellular Networks
}

\author{
Jian Song, Lam-Thanh Tu, and Marco Di Renzo \\ Paris-Saclay University - Laboratory of Signals and Systems (UMR-8506) \\ CNRS - CentraleSupélec - University Paris-Sud \\ 3 rue Joliot-Curie, 91192 Gif-sur-Yvette (Paris), France \\ e-mail: \{jian.song, lamthanh.tu, marco.direnzo\}@12s.centralesupelec.fr
}

\begin{abstract}
Recent studies have demonstrated that millimeterwave cellular networks may operate either in the noise- or in the interference-limited regime, depending on several parameters, which include the density of base stations, the density and size of obstacles/blockages, the antenna beamwidth, and the transmission bandwidth. The objective of the present paper is to exploit tools from stochastic geometry for obtaining a mathematically tractable framework that allows us to identify the operating conditions under which millimeter-wave cellular networks operate in the inference-limited regime and to assess the potential advantages of interference alignment, by taking into account the overhead cost of base station cooperation due to the estimation of channel state information.
\end{abstract}

\section{INTRODUCTION}

The use of the millimeter wave (mmWave) frequency band for application to the wireless access of heterogeneous ultradense cellular networks constitutes a promising solution for achieving the data rate that the fifth generation $(5 \mathrm{G})$ of cellular systems is expected to provide. Recent results have shown, in fact, that mmWave cellular networks may outperform their counterparts that operate at conventional frequencies, i.e., below $6 \mathrm{GHz}$, provided that the Base Stations (BSs) are sufficiently densely deployed, highly-directive antennas are used at both the BSs and the Mobile Terminals (MTs), and the transmission bandwidth is sufficiently large [1]- [4].

In [1], in particular, it is shown that mmWave cellular networks with an average cell radius of 50 meters and with a transmission bandwidth of the order of $\mathrm{GHz}$ may outperform conventional cellular networks in dense urban environments. The reason of this performance gain is that mmWave cellular networks operate close to the noise-limited regime while conventional cellular networks are interference-limited. The mathematical analysis and numerical illustrations shown in [1] have unveiled that mmWave cellular networks exhibit a noise-limited to interference-limited transitional behavior as a function of the inter-site distance of the BSs, the antenna beamwidth, and the transmission bandwidth. Stated differently, mmWave cellular networks may operate in the interferencelimited regime as well, if practical constraints on the transmission bandwidth and on the antenna beamwidth need to be imposed and, consequently, the density of BSs needs to be increased for providing an adequate coverage to the MTs.

In these application scenarios, interference management schemes need to be applied, in order to exploit the potential of mmWave cellular networks for wireless access. A promising technique for reducing the other-cell interference is Interference Alignment (IA) [5], since it is capable of best using the available degrees of freedom in interference channels. In IA, clusters of BSs, which serve different MTs on the same physical channel, cooperate and apply linear precoding methods to suppress the other-cell interference at each intended MT. If applied to interference-limited mmWave cellular networks, IA provides opportunities for suppressing the strongest interferers and enhancing the coverage and rate. The larger the number of cooperating BSs is, in general, the smaller the other-cell interference is and, hence, the better the performance is. In order to appropriately work, however, IA necessitates channel state information at the BSs. This is usually obtained with the aid of pilot signals that are transmitted before the data signals [6]- [8]. In general, in addition, the larger the number of cooperating BSs is, the longer the time for estimating the channel is. This negatively affects the achievable rate of IA.

Motivated by these considerations, we investigate the potential throughput of IA for application to mmWave cellular networks. The objective is to quantify the interference reduction vs. training overhead tradeoff of IA, as a function of the number of cooperating BSs. To this end, we exploit tools from stochastic geometry and model the BSs of mmWave cellular networks as points of a Poisson Point Process (PPP) [9]. Based on the mathematical approach introduced in [10], we develop a tractable framework that is easier to compute than numerical simulations. From it, we show that an optimal number of cooperating BSs that maximizes the potential throughput of mmWave cellular networks that use IA exists.

The remainder of the present paper is organized as follows. In Section II, the system model is introduced. In Section III, the proposed mathematical framework for the potential throughput is presented. In Section IV, the framework is validated with the aid of Monte Carlo simulations and numerical illustrations are shown in order to quantify the impact of IA as a function of the density of BSs and of the number of cooperating BSs. Finally, Section V concludes this paper.

\section{SySTEM MODEL}

\section{A. PPP-Based Cellular Networks Modeling}

We consider a downlink cellular network where the BSs are modeled as points of a homogeneous PPP, denoted by $\Psi_{\mathrm{BS}}$, of density $\lambda_{\mathrm{BS}}$. The MTs are modeled as another independent homogeneous PPP, denoted by $\Psi_{\mathrm{MT}}$, of density $\lambda_{\mathrm{MT}}$. Each BS and MT are equipped with $N_{T}$ transmit and $N_{R}$ receive 
antennas, respectively. We assume $\lambda_{\mathrm{MT}} \gg \lambda_{\mathrm{BS}}$, i.e., saturated traffic conditions hold and, thus, the BSs are all active. Each BS is assumed to emit a constant transmit power $P$.

\section{B. Channel Modeling}

A quasi-static channel model is assumed, where each BSto-MT link is characterized by a blockage probability, which takes into account whether the link is in Line-Of-Sight (LOS) or in Non-LOS (NLOS), path-loss, shadowing and fast-fading.

1) Blockage Modeling: In the mmWave frequency band, modeling blockages is of primary importance [1], [4]. A BSto-MT link is said to be in NLOS if a blockage, e.g., a building in an urban environment, is present in the straight line between the BS and the MT. Otherwise, it is in LOS. Typically, the presence of blockages and, thus, the occurrence of LOS and NLOS conditions is taken into account statistically with the aid of probability functions that depend on the distance between BSs and MTs [10]. In this paper, the LOS and NLOS probability are denoted by $p_{\mathrm{LOS}}(\cdot)$ and $p_{\mathrm{NLOS}}(\cdot)$, respectively. They are assumed to depend only on the BS-to-MT distance, $r$, and the identity $p_{\mathrm{LOS}}(r)+p_{\mathrm{NLOS}}(r)=1$ holds for every $r$.

The mathematical analysis of mmWave cellular networks in the presence of realistic blockage models is known to be intractable. To overcome this issue, we adopt the tractable model first introduced in [1] and then generalized in [10] for arbitrary blockage models in the presence of shadowing and general load conditions. The adopted blockage model is known as the multi-ball model and it is summarized as follows [3].

In the multi-ball blockage model, the distance between the typical MT and its serving BS is divided in $B+1$ annular regions, which correspond to $B$ balls whose center is the MT. Let $0=D_{0}<D_{1}<D_{2}<\cdots<D_{B}<D_{B+1}=\infty$ denote the radii of the $B$ balls. The LOS and NLOS probabilities, $p_{s}(\cdot)$ for $s \in\{\mathrm{LOS}, \mathrm{NLOS}\}$, can be formulated as follows:

$$
p_{s}(r)=\sum_{b=1}^{B+1} q_{s}^{\left[D_{b-1}, D_{b}\right]} \mathbf{1}\left(D_{b-1}<r<D_{b}\right)
$$

where $q_{s}^{[x, y]}$ for $s \in\{$ LOS, NLOS $\}$ is the probability that a link of length $r \in[x, y]$ is in state $s$ and $\mathbf{1}(\cdot)$ is the indicator function. Since a link can be only in LOS or NLOS, the equality $\sum_{s \in\{\text { LOS.NLOS }\}} q_{s}^{[x, y]}=1$ holds in every region.

2) Path-Loss Modeling: The path-loss of LOS and NLOS links is formulated as $l_{s}(r)=k_{s} r^{\beta_{s}}$ for $s \in\{\mathrm{LOS}$, NLOS $\}$, where $k_{s}$ denotes the path-loss constant, $\beta_{s}$ denotes the pathloss slope, and $r$ is the BS-to-MT distance, respectively.

3) Shadowing Modeling: Consider a link of length $r$ in state $s \in\{$ LOS, NLOS $\}$. The shadowing, denoted by $\chi_{s}$, is assumed to follow a log-normal distribution with mean $\mu_{s}$ (in $\mathrm{dB}$ ) and standard deviation $\sigma_{s}$ (in $\mathrm{dB}$ ). The probability density function of shadowing can be formulated as $f_{\chi_{s}}=$ $\frac{10}{\ln (10)} \frac{1}{\sqrt{2 \pi} \sigma_{s} x} \exp \left(-\frac{\left(10 \log _{10} x-\mu_{s}\right)^{2}}{2 \sigma_{s}^{2}}\right)$.

4) Fading Modeling: In addition to blockage, path-loss and shadowing, each BS-to-MT link is subject to fast-fading, which is modeled as an independent complex channel gain denoted by $h$. The power gain $|h|^{2}$ is assumed to follow an exponential distribution (i.e., Rayleigh fading is assumed) with mean value equal to $\Omega_{\mathrm{LOS}}=\Omega_{\mathrm{NLOS}}=1$.

\section{Directional Beamforming Modeling}

In order to overcome the large path-loss at high frequency bands, mmWave cellular networks are expected to use directional antennas for enhancing the link reliability and reducing the other-cell interference. We assume that directional beamforming is employed at both the BSs and MTs. Similar to previous works, a two-lobe model for the radiation pattern of the directional antennas is assumed [1]. The generalizations to other radiation pattern models is possible by using the approach recently introduced in [10]. Accordingly, the antenna gain of BSs and MTs can be formulated as follows:

$$
G_{q}(\theta)=\left\{\begin{array}{lll}
G_{q}^{(\mathrm{M})} & \text { if } & |\theta| \leq \frac{\omega_{q}}{2} \\
G_{q}^{(\mathrm{S})} & \text { if } & |\theta|>\frac{\omega_{q}}{2}
\end{array}\right.
$$

where $q \in\{\mathrm{BS}, \mathrm{MT}\}, \theta \in\{-\pi, \pi\}$ is the angle off the boresight direction, $\omega_{q}$ is the beamwidth of the main lobe, $G_{q}^{(\mathrm{M})}$ and $G_{q}^{(\mathrm{S})}$ are the array gains of main and side lobes.

The typical MT and its serving BS are assumed to estimate the angles of arrival and to adjust their steering directions accordingly, so that the directivity gain of the intended link is $G^{(0)}=G_{\mathrm{BS}}^{(\mathrm{M})} G_{\mathrm{MT}}^{(\mathrm{M})}$. The directivity gain of the interfering BSs, $G^{(i)}$ where $i$ identifies the $i$ th interfering BS, are assumed to be randomly oriented with respect to each other and to be independently and uniformly distributed in $[-\pi, \pi)$. Therefore, the probability density function of $G^{(i)}$ can be formulated as:

$$
f_{G^{(i)}}(g)=\sum_{m \in\{\mathrm{M}, \mathrm{S}\}} \sum_{n \in\{\mathrm{M}, \mathrm{S}\}} \frac{\omega_{\mathrm{BS}}^{(m)}}{2 \pi} \frac{\omega_{\mathrm{MT}}^{(n)}}{2 \pi} \delta\left(g-G_{\mathrm{BS}}^{(m)} G_{\mathrm{MT}}^{(n)}\right)
$$

where $\delta(\cdot)$ is the Dirac delta function, $\omega_{q}^{(\mathrm{M})}=\omega_{q}$ and $\omega_{q}^{(\mathrm{S})}=$ $2 \pi-\omega_{q}$ for $q \in\{\mathrm{BS}, \mathrm{MT}\}$.

\section{Cell Association Criterion}

The cell association is based on the highest average received power criterion, which accounts for the path-loss and shadowing while the fast-fading is neglected. We assume that the LOS or NLOS status of a BS-to-MT link is independent of the LOS or NLOS status of any other BS-to-MT link. Stated differently, no spatial correlation among the BS-to-MT links is considered. Under this assumption, the homogenous PPP of the BSs, $\Psi_{\mathrm{BS}}$, can be partitioned into two independent and inhomogeneous PPPs, $\Psi_{\mathrm{BS}, s}$ for $s \in\{\mathrm{LOS}, \mathrm{NLOS}\}$, such that $\bigcup_{s \in\{\mathrm{LOS}, \mathrm{NLOS}\}} \Psi_{\mathrm{BS}, s}=\Psi_{\mathrm{BS}}$, whose intensities are $\lambda_{\mathrm{BS}, \mathrm{LOS}}(r)=\lambda_{\mathrm{BS}} p_{\mathrm{LOS}}(r)$ and $\lambda_{\mathrm{BS}, \mathrm{NLOS}}(r)=\lambda_{\mathrm{BS}} p_{\mathrm{NLOS}}(r)$.

Let $L_{s}(r)=l_{s}(r) / \chi_{s}$ for $s \in\{$ LOS, NLOS $\}$ be the generalized path-loss that accounts for shadowing, i.e., the inverse average received power, of a BS-to-MT link of length $r$. The generalized path-loss of the serving BS of the typical MT can be formulated as $L^{(0)}=\min \left\{L_{\mathrm{LOS}}^{(0)}, L_{\mathrm{NLOS}}^{(0)}\right\}$, where $L_{s}^{(0)}$ for $s \in\{$ LOS, NLOS $\}$ is defined as follows:

$$
L_{s}^{(0)}=\min _{n \in \Psi_{\mathrm{BS}, s}}\left\{L_{s}\left(r^{(n)}\right)\right\}
$$

and $r^{(n)}$ is the distance between the $n$th BS of $\Psi_{\mathrm{BS}, s}$ and MT. 


$$
\begin{aligned}
& \widehat{\Lambda}_{s}([0, x))=\pi \widehat{\lambda}_{\mathrm{BS}, s} \sum_{b=1}^{\widehat{B}} \widehat{q}_{s}^{\left[\widehat{D}_{b-1}, \widehat{D}_{b}\right]} H\left(x-k_{s} \widehat{D}_{b-1}^{\beta_{s}}\right) \bar{H}\left(x-k_{s} \widehat{D}_{b}^{\beta_{s}}\right)\left(\left(x / k_{s}\right)^{2 / \beta_{s}}-\widehat{D}_{b-1}^{2}\right) \\
& +\pi \widehat{\lambda}_{\mathrm{BS}, s} \sum_{b=1}^{\widehat{B}} \widehat{q}_{s}^{\left.\widehat{D}_{b-1}, \widehat{D}_{b}\right]} H\left(x-k_{s} \widehat{D}_{b}^{\beta_{s}}\right)\left(\widehat{D}_{b}^{2}-\widehat{D}_{b-1}^{2}\right)+\pi \widehat{\lambda}_{\mathrm{BS}, s} \widehat{q}_{s}^{\left[\widehat{D}_{\widehat{B}}, \infty\right]} H\left(x-k_{s} \widehat{D}_{\widehat{B}}^{\beta_{s}}\right)\left(\left(x / k_{s}\right)^{2 / \beta_{s}}-\widehat{D}_{\widehat{B}}^{2}\right)
\end{aligned}
$$

\section{E. Tractable Mathematical Modeling}

Taking spatial blockages and shadowing into account makes the mathematical analysis of mmWave cellular networks intractable. To overcome this limitation, we capitalize on the approach introduced in [10], which is referred to as the Intensity Matching (IM) approach. The interested readers are referred to [10] for further information. In simple terms, the IM approach is an approximation that allows us to account for the impact of blockages and shadowing at an affordable analytical complexity. It is based on the following assumptions:

- Any blockage model, if different from the multi-ball model formulated in (1), is approximated with a multiball model whose number of balls, denoted by $\widehat{B}$, is chosen to tradeoff computational complexity and approximation accuracy. Usually three-five balls are enough to get sufficiently accurate results.

- Shadowing is taken into account as follows: i) by scaling the density of BSs in LOS and NLOS with respect to the typical MT as $\widehat{\lambda}_{\mathrm{BS}, s}=\lambda_{\mathrm{BS}} \mathbb{E}_{\chi_{s}}\left\{\chi_{s}^{2 / \beta_{s}}\right\}=$ $\lambda_{\mathrm{BS}} \exp \left(2 \frac{\bar{\mu}_{s}}{\beta_{s}}+2 \frac{\bar{\sigma}_{s}^{2}}{\beta_{s}^{2}}\right)$, where $\mathbb{E}\{\cdot\}$ is the expectation operator, and $\bar{\mu}_{s}$ and $\bar{\sigma}_{s}$ are the mean and standard deviation of log-normal shadowing expressed in neper, ii) by scaling the radii, $D_{b}$, and the occurrence probabilities, $q_{s}^{\left[D_{b-1}, D_{b}\right]}$, of the $\widehat{B}$ balls in (1). The scaled radii and probabilities are denoted by $\widehat{D}_{b}$ and $\left.\widehat{q}_{s} \widehat{D}_{b-1}, \widehat{D}_{b}\right]$

- The approximated (scaled) parameters $\widehat{B}, \widehat{D}_{b}$ and $\left.\widehat{q}_{s} \widehat{D}_{b-1}, \widehat{D}_{b}\right]$ are obtained by minimizing the mean square error between i) the intensity measure of the generalized path-loss corresponding to the exact blockage and shadowing models and to a density of BSs equal to $\lambda_{\mathrm{BS}, s}$ and ii) the intensity measure of the generalized pathloss corresponding to a multi-ball blockage model with parameters $\widehat{B}, \widehat{D}_{b}$ and $\widehat{q}_{s}^{\left[\widehat{D}_{b-1}, \widehat{D}_{b}\right]}$ and to a density of BSs equal to $\widehat{\lambda}_{\mathrm{BS}, s}$. The definition of intensity measure of inhomogeneous PPPs and the mathematical details of the matching criterion are available in [10].

With the aid of the IM approach, the set of generalized path-losses corresponding to the PPP of BSs, $\Psi_{\mathrm{BS}}$, can be shown to be an inhomogeneous PPP whose intensity measure is $\widehat{\Lambda}([0, x))=\sum_{s \in\{\mathrm{LOS}, \mathrm{NLOS}\}} \widehat{\Lambda}_{s}([0, x))$, where $\widehat{\Lambda}_{s}([\cdot, \cdot))$ is defined in (5), $H(\cdot)$ is the Heaviside function and $\bar{H}(x)=$ $1-H(x)$. Since inhomogeneous PPPs are completely characterized by their intensity measure, (5) constitutes an enabling result for the analysis of mmWave cellular networks.

\section{F. Interference Alignment}

As mentioned in Section I, ultra-dense mmWave cellular networks may be interference-limited. Thus, allowing the cooperation among BSs may reduce the impact of strong interferers. As an example of BSs cooperation for interference reduction, we analyze IA. For simplicity, we assume that a single information stream is transmitted. As far as the typical MT is concerned, in particular, IA operates as follows [6]- [8]. First, the $K$ BSs providing the smallest $K$ generalized path-losses to the typical MT are identified. The smallest generalized path-loss is denoted by $L^{(0)}$, while the $k$ th smallest generalized path-loss is denoted by $L^{(k)}$ for $k=2,3, \ldots, K-1$. Then, the $K$ cooperating BSs apply linear precoding and decoding vectors in order to suppress the othercell interference generated by the $K-1$ interfering BSs for the typical MT. The mathematical formulation for obtaining precoding and decoding vectors is available in [5]. We use the min-leakage algorithm for obtaining them [6]- [8]. The BSs outside the cluster of $K$ cooperating BSs, on the other hand, still create other-cell interference towards the typical MT. It is worth noting that the number of cooperating BSs $K$ needs to be chosen according to the feasibility conditions:

$$
N_{T}+N_{R} \geq(K+1) \text { and } \min \left\{N_{T}, N_{R}\right\} \geq 2
$$

In order to compute the precoding and decoding vectors, the cooperating BSs necessitate channel state information of the links inside the cluster. To obtain estimates of the BS-toMT channel gains inside the cluster of $K$ cooperating BSs, we adopt the pilot overhead model in [7] for application to frequency-division duplex systems. In simple terms, a blockfading channel model is assumed, where the block-length expressed in terms of multiple of the symbol time, $N_{\mathrm{b}}$, depends on the Doppler spread $f_{D}$, i.e., $N_{\mathrm{b}}=\frac{1}{2 f_{D}}$. According to the pilot overhead model in [7], the number of pilot symbols, $N_{\mathrm{p}}$, that are needed for channel estimation is $N_{\mathrm{p}}=N_{\mathrm{pf}}+N_{\mathrm{pr}}+$ $N_{\mathrm{fb}}+N_{\text {pd }}$, where $N_{\mathrm{pf}} \geq K N_{T}, N_{\mathrm{pr}} \geq K N_{R}, N_{\mathrm{fb}} \geq K^{2} N_{T}$ and $N_{\mathrm{pd}} \geq K$. As a result, the number of symbols available for data transmission is $N_{\mathrm{d}}=N_{\mathrm{b}}-N_{\mathrm{p}}$. Since a finite number of pilots are used, in addition, the channels are estimated with some errors, which does not permit perfect interference suppression inside the cluster of $K$ cooperating BSs. For further details, the interested readers are referred to [6]- [8]. In the rest of the present paper, we assume $N_{\mathrm{pf}}=K N_{T}$, $N_{\mathrm{pr}}=K N_{R}, N_{\mathrm{fb}}=K^{2} N_{T}$ and $N_{\mathrm{pd}}=K$.

\section{System-Level Performance Evaluation}

In this section, we provide a tractable mathematical framework for computing the Potential Throughput (PT) of mmWave cellular networks with IA. The PT is defined as:

$$
\mathrm{PT}=\tau \log _{2}\left(1+\gamma_{\mathrm{th}}\right) \operatorname{Pr}\left\{\mathrm{SINR} \geq \gamma_{\mathrm{th}}\right\}
$$

where $\tau=\frac{N_{\mathrm{b}}-\left(N_{\mathrm{pf}}+N_{\mathrm{pr}}+N_{\mathrm{fb}}+N_{\mathrm{pd}}\right)}{N_{\mathrm{b}}}$ accounts for the effective resources used for data transmission and, thus, takes the pilot 


$$
\begin{aligned}
& \mathrm{PT}=\tau \log _{2}\left(1+\gamma_{\mathrm{th}}\right) \frac{\Omega_{\widetilde{u}^{(0)}}}{\Omega_{\widetilde{u}^{(}(0)}+\gamma_{\mathrm{th}} \Omega_{\widetilde{u}^{(0)}}}
\end{aligned}
$$

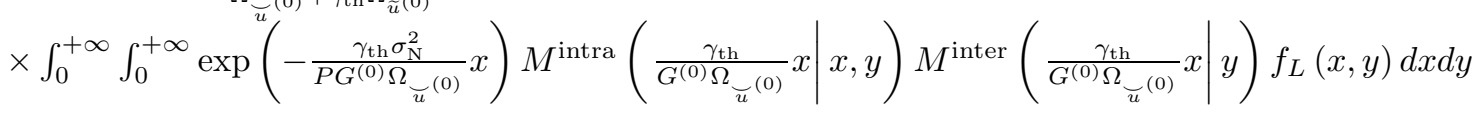

$$
\begin{aligned}
\Upsilon_{s}(\Omega, z \mid u) & =\pi \widehat{\lambda}_{\mathrm{BS}, s}\left(\frac{u}{k_{s}}\right)^{\frac{2}{\beta_{s}}} \Theta_{s}\left(\frac{\Omega}{u}, z\right) \sum_{b=1}^{\widehat{B}} \widehat{q}_{s}^{\left[\widehat{D}_{b-1}, \widehat{D}_{b}\right]} \bar{H}\left(u-k_{s} \widehat{D}_{b}^{\beta_{s}}\right) H\left(u-k_{s} \widehat{D}_{b-1}^{\beta_{s}}\right) \\
& +\pi \widehat{\lambda}_{\mathrm{BS}, s} \sum_{b=1}^{\widehat{B}} \widehat{q}_{s}^{\left[\widehat{D}_{b-1}, \widehat{D}_{b}\right]}\left(\widehat{D}_{b-1}^{2} \Theta_{s}\left(\frac{\Omega}{k_{s} \widehat{D}_{b-1}^{\beta_{s}}}, z\right) \bar{H}\left(u-k_{s} \widehat{D}_{b-1}^{\beta_{s}}\right)-\widehat{D}_{b}^{2} \Theta_{s}\left(\frac{\Omega}{k_{s} \widehat{D}_{b}^{\beta_{s}}}, z\right) \bar{H}\left(u-k_{s} \widehat{D}_{b}^{\beta_{s}}\right)\right) \\
& +\pi \widehat{\lambda}_{\mathrm{BS}, s} \widehat{q}_{s}^{\left.\widehat{D}_{\widehat{B}}, \infty\right]}\left(\widehat{D}_{\widehat{B}}^{2} \Theta_{s}\left(\frac{\Omega}{k_{s} \widehat{D}_{\widehat{B}}^{\beta_{s}}}, z\right) \bar{H}\left(u-k_{s} \widehat{D}_{\widehat{B}}^{\beta_{s}}\right)+\left(\frac{u}{k_{s}}\right)^{\frac{2}{\beta_{s}}} \Theta_{s}\left(\frac{\Omega}{u}, z\right) H\left(u-k_{s} \widehat{D}_{\widehat{B}}^{\beta_{s}}\right)\right)
\end{aligned}
$$

training overhead into account, $\gamma_{\text {th }}$ is the decoding threshold, and SINR is the Signal-to-Interference+Noise-Ratio.

Based on the system model introduced in Section II, the SINR of mmWave cellular networks with IA can be formulated as follows (the details are omitted for brevity):

$$
\mathrm{SINR}=\frac{\left(P G^{(0)} / L^{(0)}\right) \breve{u}^{(0)}}{\left(P G^{(0)} / L^{(0)}\right) \widetilde{u}^{(0)}+\sigma_{\mathrm{N}}^{2}+P I_{\mathrm{agg}}\left(L^{(0)}, L^{(K-1)}\right)}
$$

where $\sigma_{\mathrm{N}}^{2}$ is the noise power, $I_{\mathrm{agg}}\left(L^{(0)}, L^{(K-1)}\right)=$ $I_{\text {agg }}^{\text {intra }}\left(L^{(0)}, L^{(K-1)}\right)+I_{\text {agg }}^{\text {inter }}\left(L^{(K-1)}\right)$ is the aggregate othercell interference, where $I_{\text {agg }}^{\text {intra }}(\cdot, \cdot)$ originates from the $K$ cooperating BSs and is due to channel estimation errors and $I_{\text {agg }}^{\text {inter }}(\cdot)$ originates from all the other non-cooperating BSs, $\breve{u}^{(0)}$ is the equivalent channel gain of the intended link after decoding, and $\widetilde{u}^{(0)}$ is the equivalent channel gain of the intended link after decoding due to channel estimation errors.

With the aid of some algebra, the following can be proved:

- $\breve{u}^{(0)}$ is an exponential random variable with mean

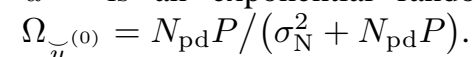

- $\widetilde{u}^{(0)}$ is an exponential random variable with mean $\Omega_{\widetilde{u}^{(0)}}=$ $\sigma_{\mathrm{N}}^{2} /\left(\sigma_{\mathrm{N}}^{2}+N_{\mathrm{pd}} P\right) \cdot \breve{u}^{(0)}$ and $\widetilde{u}^{(0)}$ are independent.

- $I_{\text {agg }}^{\text {intra }}(\cdot, \cdot)$ and $I_{\text {agg }}^{\text {inter }}(\cdot)$ can be formulated as follows:

$$
\begin{aligned}
I_{\mathrm{agg}}^{\text {intra }} & \left(L^{(0)}, L^{(K-1)}\right) \\
= & \sum_{k=1}^{K-1} \frac{G^{(k)}}{L^{(k)}} \widetilde{g}^{(k)} \mathbf{1}\left(L^{(0)}<L^{(k)}<L^{(K-1)}\right) \\
I_{\mathrm{agg}}^{\text {inter }} & \left(L^{(K-1)}\right) \\
= & \sum_{i \in \Psi_{\mathrm{BS}}} \frac{G^{(i)}}{L^{(i)}}\left(\breve{g}^{(i)}+\widehat{g}^{(i)}\right) \mathbf{1}\left(L^{(i)}>L^{(K-1)}\right)
\end{aligned}
$$

where $\widetilde{g}^{(k)}, \breve{g}^{(i)}$ and $\widehat{g}^{(i)}$ are independent and exponentially distributed random variables with means $\Omega_{\widetilde{g}^{(k)}}=$ $\Omega_{g}, \Omega_{{ }^{(i)}}=1-\Omega_{g}$ and $\Omega_{g^{(i)}}=\Omega_{g}$, respectively, where:

$$
\begin{aligned}
\Omega_{g}=\frac{N_{T} \sigma_{\mathrm{N}}^{2}}{N_{\mathrm{pf}} P} & +\frac{\sigma_{\mathrm{N}}^{2}}{\left(K N_{T}-N_{R}\right) P} \\
& \times\left(\frac{N_{R}^{2}}{N_{\mathrm{pr}}}+\frac{K N_{T} N_{R}}{N_{\mathrm{fb}}}\left(1+\frac{N_{R} \sigma_{\mathrm{N}}^{2}}{N_{\mathrm{pr}} P}\right)\right)
\end{aligned}
$$

We note that, with respect to currently available mathematical frameworks for the analysis of cellular networks by using stochastic geometry [10], the main difference of the problem formulation in (8) is the presence of additional terms that account for channel estimation errors and for the dependence of the aggregate interference on both the smallest generalized path-loss $L^{(0)}$ and on the $(K-1)$ th smallest generalized pathloss $L^{(K-1)}$, which are correlated random variables.

By using the same line of thought as in [10], a mathematically tractable and computable expression of the PT is provided in (12), where $f_{L}(\cdot, \cdot)$ is the joint probability density function of $L^{(0)}$ and $L^{(K-1)}, M^{\text {intra }}(\cdot \mid \cdot, \cdot)$ is the Laplace transform of $I_{\text {agg }}^{\text {intra }}(\cdot, \cdot)$ conditioned on $L^{(0)}$ and $L^{(K-1)}$, and $M^{\text {inter }}(\cdot \mid \cdot)$ is the Laplace transform of $I_{\text {agg }}^{\text {inter }}(\cdot)$ conditioned on $L^{(K-1)}$. The function $f_{L}(\cdot, \cdot)$ can be formulated as:

$$
\begin{array}{r}
f_{L}(x, y)=\frac{(\widehat{\Lambda}([0, y))-\widehat{\Lambda}([0, x)))^{K-2}}{(K-2) !} \exp (-\widehat{\Lambda}([0, y))) \\
\times \widehat{\Lambda}^{(1)}([0, x)) \widehat{\Lambda}^{(1)}([0, y)) H(y-x)
\end{array}
$$

where $\widehat{\Lambda}^{(1)}([\cdot, \cdot))$ is the first-order derivative of $\widehat{\Lambda}([\cdot, \cdot))$ in (5), which can be explicitly expressed as $\widehat{\Lambda}^{(1)}([0, x))=$ $\sum_{s \in\{\operatorname{LOS}, \mathrm{NLOS}\}} \widehat{\Lambda}_{s}^{(1)}([0, x))$, where $\widehat{\Lambda}_{s}^{(1)}([\cdot, \cdot))$ is:

$$
\begin{aligned}
& \widehat{\Lambda}_{s}^{(1)}([0, x))=\pi \widehat{\lambda}_{\mathrm{BS}, s}\left(2 / \beta_{s}\right) k_{s}^{-2 / \beta_{s}} x^{2 / \beta_{s}-1} \\
& \times \sum_{b=1}^{\widehat{B}} \widehat{q}_{s}^{\left[\widehat{D}_{b-1}, \widehat{D}_{b}\right]} \bar{H}\left(x-k_{s} \widehat{D}_{b}^{\beta_{s}}\right) H\left(x-k_{s} \widehat{D}_{b-1}^{\beta_{s}}\right) \\
& +\pi \widehat{\lambda}_{\mathrm{BS}, s}\left(2 / \beta_{s}\right) k_{s}^{-2 / \beta_{s}} x^{2 / \beta_{s}-1} \widehat{q}_{s}^{\left[\widehat{D}_{\widehat{B}}, \infty\right]} H\left(x-k_{s} \widehat{D}_{\hat{B}}^{\beta_{s}}\right)
\end{aligned}
$$

and $M^{\text {intra }}(\cdot \mid \cdot, \cdot)$ and $M^{\text {inter }}(\cdot \mid \cdot)$ can be formulated as:

$$
\begin{aligned}
& M^{\text {intra }}(z \mid x, y)=\exp \left(\sum_{s \in\{\text { LOS }, \text { NLOS }\}} m_{s}^{\text {intra }}(z \mid x, y)\right) \\
& M^{\text {inter }}(z \mid y)=\exp \left(\sum_{s \in\{\text { LOS }, \text { NLOS }\}} m_{s}^{\text {inter }}(z \mid y)\right)
\end{aligned}
$$

where $m_{s}^{\text {intra }}(z \mid x, y)=\Upsilon_{s}\left(\Omega_{g}, z \mid x\right)-\Upsilon_{s}\left(\Omega_{g}, z \mid y\right)$, $m_{s}^{\text {inter }}(z \mid y)=\Upsilon_{s}\left(\left(1-\Omega_{g}\right), z \mid y\right)+\Upsilon_{s}\left(\Omega_{g}, z \mid y\right), \Upsilon_{s}(\cdot, \cdot \mid \cdot)$ is defined in (16) and:

$$
\begin{aligned}
\Theta_{s}(\Omega, z)= & 1-\sum_{m \in\{\mathrm{M}, \mathrm{S}\}} \sum_{n \in\{\mathrm{M}, \mathrm{S}\}} \frac{\omega_{\mathrm{BS}}^{(m)}}{2 \pi} \frac{\omega_{\mathrm{MT}}^{(m)}}{2 \pi} \\
& \times{ }_{2} F_{1}\left(-\frac{2}{\beta_{s}}, 1,1-\frac{2}{\beta_{s}},-\Omega G_{\mathrm{BS}}^{(m)} G_{\mathrm{MT}}^{(n)} z\right)
\end{aligned}
$$

\section{Numerical AND Simulation RESUlts}

In this section, the accuracy of the proposed mathematical framework is validated with the aid of Monte Carlo simulations and the feasibility of IA for enhancing the PT of mmWave cellular networks is studied. The following setup is 


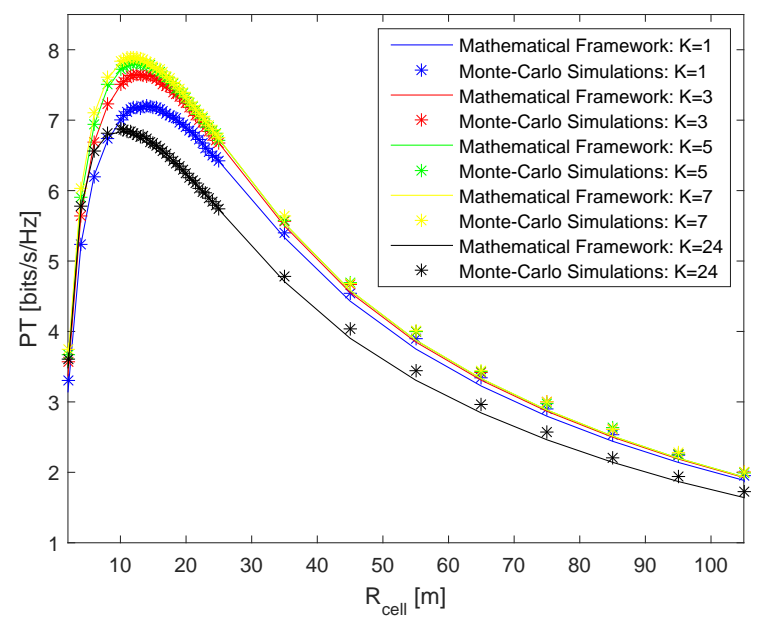

Fig. 1. PT as a function of the density of BSs $\left(\lambda_{\mathrm{BS}}=\frac{1}{\pi R_{\mathrm{cell}}^{2}}\right.$, where $R_{\text {cell }}$ is the average radius in meter) and of the number of cooperating BSs $(K)$.

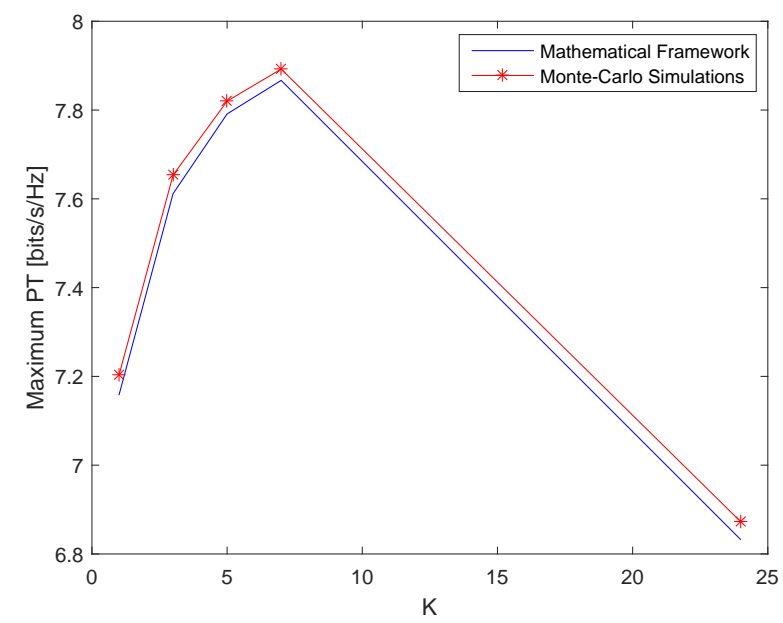

Fig. 2. Maximum PT as a function of the cluster size $K$. This figure is obtained from Fig. 1 by choosing the best $R_{\text {cell }}$ for every $K$.

considered: i) the carrier frequency is $28 \mathrm{GHz}$, ii) $P=30$ $\mathrm{dBm}$, iii) $\sigma_{\mathrm{N}}^{2}=-174+10 \log _{10}\left(B_{w}\right)+\mathcal{F}_{\mathrm{dB}}$, where $B_{w}=$ $0.5 \mathrm{GHz}$ is the bandwidth and $\mathcal{F}_{\mathrm{dB}}=10 \mathrm{~dB}$ is the noise figure, iv) $\beta_{\mathrm{LOS}}=2.1, \beta_{\mathrm{NLOS}}=2.92, k_{\mathrm{LOS}}=61.4 \mathrm{~dB}$ and $k_{\mathrm{NLOS}}=72 \mathrm{~dB}$ correspond to a mmWave channel at $28 \mathrm{GHz}$ [2], v) a blockage model based on random shape theory, i.e., $p_{\mathrm{LOS}}(r)=a_{\mathrm{RS}} \exp \left(-b_{\mathrm{RS}} r\right)$, with parameters $a_{\mathrm{RS}}=1, b_{\mathrm{RS}}=0.046$ meter $^{-1}$ is considered [10], vi) $\mu_{\mathrm{LOS}}=\mu_{\mathrm{NLOS}}=0 \mathrm{~dB}, \sigma_{\mathrm{LOS}}=5.8 \mathrm{~dB}$ and $\sigma_{\mathrm{NLOS}}=8.7$ $\mathrm{dB}$, vii) $G_{\mathrm{BS}}^{(\mathrm{M})}=G_{\mathrm{MT}}^{(\mathrm{M})}=20 \mathrm{~dB}, G_{\mathrm{BS}}^{(\mathrm{S})}=G_{\mathrm{MT}}^{(\mathrm{S})}=-10 \mathrm{~dB}$, and $\omega_{\mathrm{BS}}=\omega_{\mathrm{MT}}=30$ degrees, viii) $N_{T}=64$ and $N_{R}=2$, and ix) $f_{D}=\frac{\nu_{\mathrm{MT}}}{\text { wavelength } \times B_{w}}$, where $\nu_{\mathrm{MT}}=50$ kilometer/hour is the velocity of the MTs. The decoding threshold is $\gamma_{\mathrm{th}}=30 \mathrm{~dB}$, which maximizes the PT for the considered setup.

The mathematical framework is obtained by approximating the random shape blockage model with log-normal shadowing with a 3-ball $(\widehat{B}=3)$ blockage model, as discussed in Section II and using the IM approach. The resulting parameters are: $\widehat{D}_{1}=4.7139, \widehat{D}_{2}=18.6449, \widehat{D}_{3}=67.7784, \widehat{q}_{\mathrm{LOS}}^{\left[0, \widehat{D}_{1}\right)}=$ $0.8089, \widehat{q}_{\mathrm{LOS}}^{\left[\widehat{D}_{1}, \widehat{D}_{2}\right]}=0.3680, \widehat{q}_{\mathrm{LOS}}^{\left[\widehat{D}_{2}, \widehat{D}_{3}\right]}=0.0589, \widehat{q}_{\mathrm{LOS}}^{\left[\widehat{D}_{3}, \infty\right)}=0$.

In Fig. 1, we illustrate the PT as a function of the density of BSs and of the number of cooperating BSs. We observe that the proposed mathematical framework is in good agreement with Monte Carlo simulations. Due to the presence of blockages [10], an optimal deployment density for the BSs exists. It depends, in general, on the number of cooperating BSs $K$. As far as the impact of IA is concerned, a too large cluster size, $K$, may lead to a considerable reduction of the PT because of the associated training overhead for channel estimation. An optimal value of $K$ that maximizes the PT, in general, exists. In Fig. 2, in particular, we provide the maximum PT with respect to the density of BSs and as a function of $K$. We conclude that, in the considered setup, $K=7$ corresponds to the optimal number of cooperating BSs, which optimally tradeoffs interference reduction and channel estimation overhead. The maximum gain originating from IA is of the order of $0.7 \mathrm{bits} / \mathrm{s} / \mathrm{Hz}$.

\section{CONCLUSION}

We have proposed a tractable mathematical framework for estimating the PT of mmWave cellular networks with IA. Our study shows that: i) due to the training overhead for channel estimation, an optimal number of cooperating BSs exists and ii) due to the presence of blockages, an optimal density of the BSs exists. The proposed mathematical approach is in good agreement with Monte Carlo simulations and provides a tractable tool for optimizing mmWave cellular networks.

\section{ACKNOWLEDGMENT}

This work is supported in part by the European Commission through the H2020-ETN-5Gaura project under grant 675806 and the H2020-ETN-5Gwireless project under grant 641985.

\section{REFERENCES}

[1] M. Di Renzo, "Stochastic geometry modeling and analysis of multi-tier millimeter wave cellular networks", IEEE Trans. Wireless Commun., vol. 14, no. 9, pp. 5038-5057, Sep. 2015.

[2] W. Lu and M. Di Renzo, "Stochastic geometry modeling of mmWave cellular networks: Analysis and experimental validation", IEEE Int. Work. Measurements \& Networking , pp. 1-4, Oct. 2015.

[3] W. Lu and M. Di Renzo, "Stochastic geometry modeling of cellular networks: Analysis, simulation and experimental validation", ACM MSWiM, pp. 179-188, Nov. 2015.

[4] W. Lu and M. Di Renzo, "Millimeter wave cellular networks: Stochastic geometry modeling, analysis and experimental validation", mmWave Massive MIMO - A Paradigm for 5G, Elsevier, Nov. 2016.

[5] V. Cadambe and S. Jafar, "Interference alignment and degrees of freedom of the K-user interference channel", IEEE Trans. Inf. Theory, vol. 54, no. 8, pp. 3425-3441, Aug. 2008.

[6] O. E. Ayach, A. Lozano, and R. W. Heath. Jr., "On the overhead of interference alignment: Training, feedback, cooperation", IEEE Trans. Wireless Commun., vol. 11, no. 11, pp. 4192-4203, Nov. 2012.

[7] R. K. Mungara, G. George, and A. Lozano, "Overhead and spectral efficiency of pilot-assisted interference alignment in time-selective fading channels", IEEE Trans. Wireless Commun., vol. 13, no. 9, pp. 48844895, Sep. 2014.

[8] R. K. Mungara, D. Morales-Jimenez, and A. Lozano, "System-level Performance of interference alignment", IEEE Trans. Wireless Commun., vol. 14, no. 2, pp. 1060-1070, Feb. 2015.

[9] M. Di Renzo, A. Guidotti, and G. E. Corazza, "Average rate of downlink heterogeneous cellular networks over generalized fading channels - A stochastic geometry approach", IEEE Trans. Commun., vol. 61, no. 7, pp. 3050-3071, July 2013.

[10] M. D. Renzo, W. Lu, and P. Guan, "The intensity matching approach: A tractable stochastic geometry approximation to system-level analysis of cellular networks", IEEE Trans. Wireless Commun., vol. 15, no. 9, pp. 5963-5983, Sep. 2016. 